\title{
The Arabidopsis homologs of CCR4-associated factor 1 show mRNA deadenylation activity and play a role in plant defence responses
}

Wenxing Liang ${ }^{1,2, *}$, Changbao $\mathrm{Li}^{1,3,{ }^{*}}$, Fang Liu ${ }^{1,2}$, Hongling Jiang ${ }^{1}$, Shuyu $\mathrm{Li}^{1,2}$, Jiaqiang Sun ${ }^{1}$, Xiaoyan $\mathrm{Wu}^{1}$, Chuanyou $\mathrm{Li}^{1}$

${ }^{I}$ State Key Laboratory of Plant Genomics, National Centre for Plant Gene Research, Institute of Genetics and Developmental Biology, Chinese Academy of Sciences, Beijing 100101, China; ${ }^{2}$ Graduate School of Chinese Academy of Sciences, Beijing 100039, China; ${ }^{3}$ The State Key Laboratory of Crop Biology, Agronomy College, Shandong Agricultural University, Taian 271018, China

Messenger RNA (mRNA) turnover in eukaryotic cells begins with shortening of the poly (A) tail at the 3' end, a process called deadenylation. In yeast, the deadenylation reaction is predominantly mediated by CCR4 and CCR4associated factor 1 (CAF1), two components of the well-characterised protein complex named CCR4-NOT. We report here that $A t C A F 1 a$ and $A t C A F 1 b$, putative Arabidopsis homologs of the yeast $C A F 1$ gene, partially complement the growth defect of the yeast caf1 mutant in the presence of caffeine or at high temperatures. The expression of $A t$ $C A F 1 a$ and $A t C A F 1 b$ is induced by multiple stress-related hormones and stimuli. Both AtCAF1a and AtCAF1b show deadenylation activity in vitro and point mutations in the predicted active sites disrupt this activity. T-DNA insertion mutants disrupting the expression of $A t C A F 1 a$ and/or $A t C A F 1 b$ are defective in deadenylation of stress-related mRNAs, indicating that the two AtCAF1 proteins are involved in regulated mRNA deadenylation in vivo. Interestingly, the single and double mutants of $A t C A F 1 a$ and $A t C A F 1 b$ show reduced expression of pathogenesis-related (PR) genes $P R 1$ and $P R 2$ and are more susceptible to Pseudomonas syringae pv tomato DC3000 (Pst DC3000) infection, whereas transgenic plants over-expressing $A t C A F 1 a$ show elevated expression of $P R 1$ and $P R 2$ and increased resistance to the same pathogen. Our results suggest roles of the AtCAF1 proteins in regulated mRNA deadenylation and defence responses to pathogen infections.

Keywords: CCR4-associated factor 1 (CAF1), mRNA deadenylation, poly (A) tails, defence response, Arabidopsis thaliana Cell Research (2009) 19:307-316. doi: 10.1038/cr.2008.317; published online 9 December 2008

\section{Introduction}

Proper regulation of gene expression is crucial for essentially all biological processes [1]. Although gene expression could be regulated at different steps, controlled RNA decay is an essential process that allows rapid changes in a cell's gene expression profile, especially in response to environmental signals. In both mammalian and yeast cells, messenger RNA (mRNA) degradation usually begins with the shortening of the poly (A) tail

\footnotetext{
*These two authors contributed equally to this work.

Correspondence: Chuanyou Li

Tel: +86-10-64865313; Fax: 86-10-64873428

E-mail: cyli@genetics.ac.cn

Received 25 August 2008; revised 1 September 2008; accepted 4 September 2008; published online 9 December 2008
}

at the $3^{\prime}$ end of the mRNA (deadenylation) by a variety of deadenylases [2]. The deadenylated mRNA can then enter one of two decay pathways. In the first pathway, a complex consisting of the decapping enzymes DCP1 and DCP2 recognises the deadenylated mRNA and cleaves the $5^{\prime}$ cap, after which the XRN1 exoribonuclease hydrolyses the RNA body from its $5^{\prime}$ end. In the other pathway, deadenylated mRNAs can be degraded from the $3^{\prime}$ end by the cytoplasmic exosome complex [2]. In both cases, however, deadenylation is the initial and probably the rate-limiting step of mRNA turnover. Accumulating evidence indicates that, at least in yeast, deadenylation represents a central control point of mRNA abundance [3].

Three enzyme complexes, CCR4-NOT [4], PAN2PAN3 [5, 6] and PARN [7-9], have been identified as mRNA deadenylases in eukaryotic cells. CCR4-associat- 
ed factor 1 (CAF1) is a subunit of the CCR4-NOT complex, which is an evolutionarily conserved protein complex. The CCR4-NOT complex is involved in the control of diverse aspects of transcription and mRNA metabolism, including mRNA deadenylation and its subsequent degradation $[4,10]$. In yeast, the complex consists of at least nine core subunits, including CCR4, CAF1, CAF40, CAF130, and five NOT proteins (NOT1-NOT5) [4, 1113]. It has been shown that the CCR4 and CAF1 proteins are associated physically with each other and serve as the major cytoplasmic deadenylases in yeast cells [14-16]. However, the biochemical and physiological functions of CAF1 proteins are not clearly established. CAF1 proteins belong to the DEDDh subgroup of the DEDD family of nucleases, which requires three aspartates (D), a glutamate (E), and a nearby histidine for activity (DEDDh) $[17,18]$. Although the yeast CAF1 shows deadenylase activity in vitro $[17,19]$, the role of this activity in vivo is unclear. Inactivation of the predicted key catalytic active sites of yeast CAF1 did not affect in vivo deadenylation function [20]. Although a cafl deletion reduces the rate of in vivo poly (A) shortening [14, 17], over-expression of CCR4 can complement this defect [16]. These data support the notion that CCR4 is the principal deadenylase of the yeast CCR4-NOT complex, and a major role of CAF1 is to link CCR4 to the remainder of the CCR4NOT complex [12]. However, a recent comprehensive structure-function analysis provided evidence showing that, in addition to its contact with CCR4, the yeast CAF1, like its animal counterparts, plays important roles in mRNA deadenylation [21].

The important role of CAF1 has also been exemplified by phenotypic analyses of loss-of-function mutants of CAF1 in yeast and animals. For example, yeast caf1 mutants are hypersensitive to high temperature and caffeine $[22,23]$. Mutant male mice that lack CAF1 function are sterile [24]. Loss-of-function of CAF1 in Caenorhabditis elegans causes early embryonic and larval lethality [25].

Relatively less is known about the biochemical and physiological roles of the $C A F 1$ genes in plants. One indication comes from the observation that over-expression of the pepper $C A F 1$ gene in tomato plants confers abnormal plant growth and altered pathogen resistance [26], suggesting roles for CAF1 in both plant development and defence responses. It was recently shown that an Arabidopsis CAF1-like gene is rapidly induced after mechanical wounding [27].

Here, we show that AtCAF1a and AtCAF1b, putative Arabidopsis homologs of the yeast CAF1 protein, exhibit deadenylation activity and act redundantly in regulated deadenylation of stress-responsive mRNAs. We also provide evidence showing that over-expression or reduced expression of AtCAF1a and $A t C A F 1 b$ affects plant defence responses to pathogen infection.

\section{Results}

Hormone- and stress-induced expression of AtCAFla and $A t C A F 1 b$

AtCAFla (At3g44260) and AtCAF1b (At5g22250), which encode putative Arabidopsis homologs of the yeast CAF1 protein, were identified as jasmonic acid (JA)-inducible genes in our microarray analyses using the Arabidopsis whole genome chip (Affymetrix) [28]. Further RNA gel blot analysis indicated that, in addition to JA, the expression of the two genes was also strongly and transiently induced by abscisic acid (ABA), 1-aminocyclopropane-1-carboxylic acid (ACC, a precursor of ethylene), salicylic acid (SA), mechanical wounding, and pathogen (Pseudomonas syringae pv tomato DC3000 (Pst DC3000)) infection. As shown in Figure 1, the transcript levels of AtCAF1a and AtCAF1b peaked 15 min after these treatments and decreased rapidly thereafter. The quick and transient induction of AtCAF1a and $A t C A F 1 b$ expression by these stress-related hormones and stimuli suggested that these two genes might be associated with plant responses to biotic or abiotic stresses.

Reverse transcription (RT)-PCR analyses indicated that both $A t C A F 1 a$ and $A t C A F 1 b$ were expressed throughout the wild-type plant organs, including roots, stems, leaves, flowers and siliques (Figure 2A). The highest expression level of the two AtCAF1 genes was found in leaves, followed by roots and siliques, with relatively low expression in stems and flowers (Figure 2A).
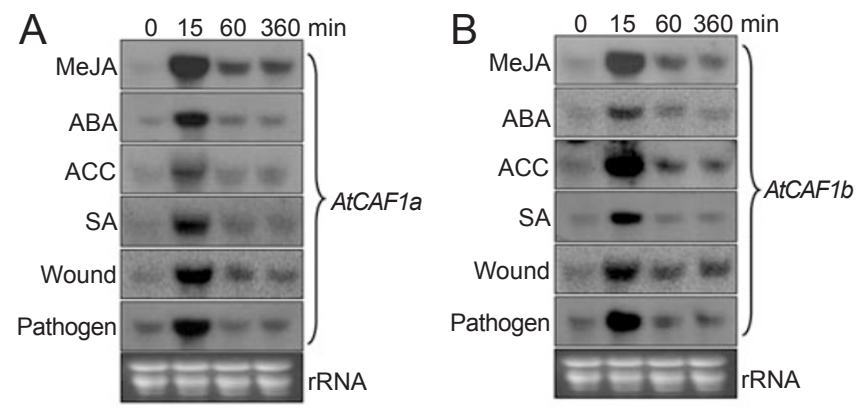

Figure 1 RNA gel blot analysis of AtCAF1a (A) and AtCAF1b (B) expression in response to different treatments. Two-week-old Col-0 plants were treated with $50 \mu \mathrm{M}$ MeJA, $20 \mu \mathrm{M}$ ABA, $50 \mu \mathrm{M}$ ACC and $20 \mu \mathrm{M} \mathrm{SA}$, wounded with a haemostat (Wound), or infected with Pst DC3000 (Pathogen). Leaves were harvested for RNA extraction at the indicated times after treatment. Each lane was loaded with $20 \mu \mathrm{g}$ of total RNA. Ethidium bromide staining of rRNA served as a loading control. 
A

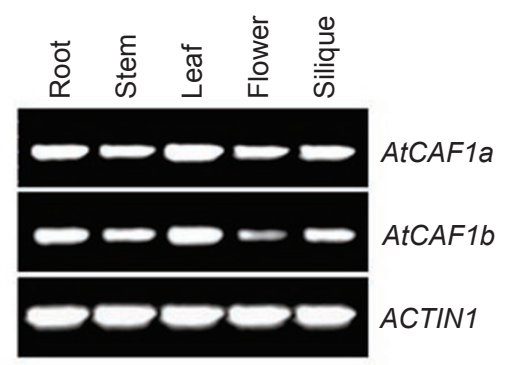

B

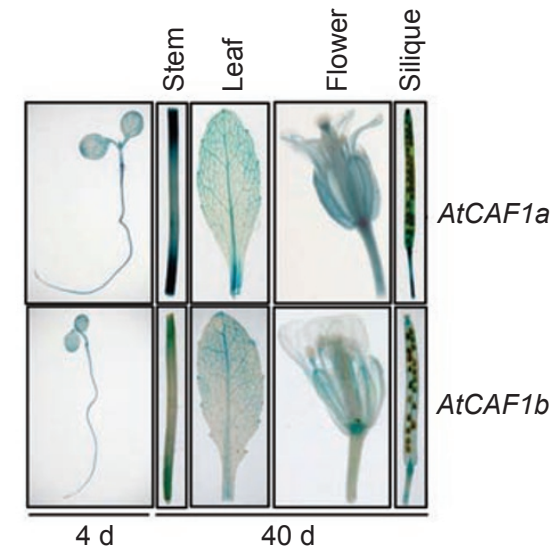

Figure 2 Spatial expression patterns of the AtCAF1 genes. (A) Expression of the two AtCAF1 genes in different organs revealed by RT-PCR analysis. Amplification of the ACTIN1 gene served as a control. (B) Promoter-driven GUS expression patterns of the two AtCAF1 genes. Shown are GUS staining of 4-dold seedlings and organs from 40-d-old plants.

The tissue-specific expression patterns of these genes were also investigated with transgenic plants expressing the glucuronidase (GUS) reporter under the control of the AtCAF1 promoters. GUS activities were detected in stems, leaves, floral parts and siliques (Figure 2B), confirming that the $A t C A F 1$ genes are ubiquitously expressed in different organs.

AtCAF1 genes partially complement the phenotypes of the yeast cafl mutant

Two of the most prominent phenotypes of the yeast caf1 mutants are their temperature and caffeine sensitivities $[22,23]$. To examine the functional properties of the AtCAF1 genes, the two genes were heterologously expressed in the yeast caf1 mutant strain KY803-c1. As shown in Figure 3, the constructs pYES2-AtCAF1a and pYES2-AtCAF1b partially restored colony formation of the cafl mutant at $37{ }^{\circ} \mathrm{C}$ or on a YD plate containing 5 $\mathrm{mM}$ caffeine, but the vector control could not restore colony formation, indicating that AtCAF1a and AtCAF1b represent the Arabidopsis homologs of the yeast CAF1 protein.

AtCAF1 proteins exhibit $3^{\prime}-5^{\prime}$ exonuclease activity in vitro

The high sequence similarity of AtCAF1 proteins to those from other eukaryotic organisms, and the conservation of the DEDDh amino acids required for deadenylation activity (Supplementary information, Figure S1), suggested that they may function as deadenylases. To test the role of AtCAF1a in deadenylation, a recombinant His-AtCAF1a fusion protein was expressed in Escherichia coli. The purified His-AtCAF1a fusion protein was incubated with poly (A) as described in Materials and Methods. Time-dependent shortening of the $5^{\prime}$-labelled poly (A) indicated that AtCAF 1a is a functional 3' -5 ' exonuclease

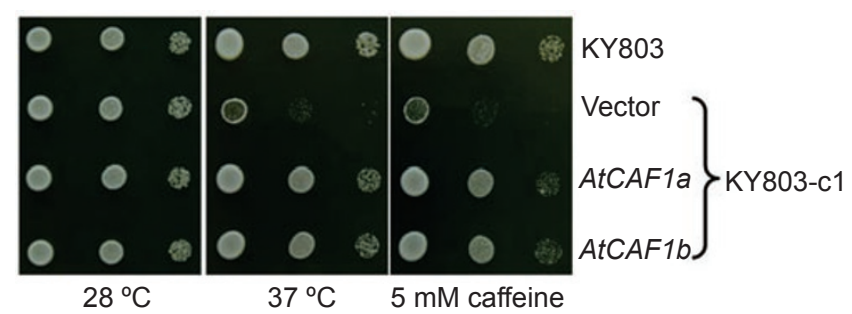

Figure 3 Effects of heterologous expression of AtCAF1 genes on the growth of the yeast caf1 mutant. Yeast strains KY803 and KY803-c1 with pYES2-AtCAF1a, pYES2-AtCAF1b or with pYES2 vector were grown on a $Y D$ plate at $28^{\circ} \mathrm{C}$, on a $Y D$ plate at $37{ }^{\circ} \mathrm{C}$ or on a YD plate with $5 \mathrm{mM}$ caffeine. In each panel, from left to right, 20 -fold fewer cells were plated in each column.

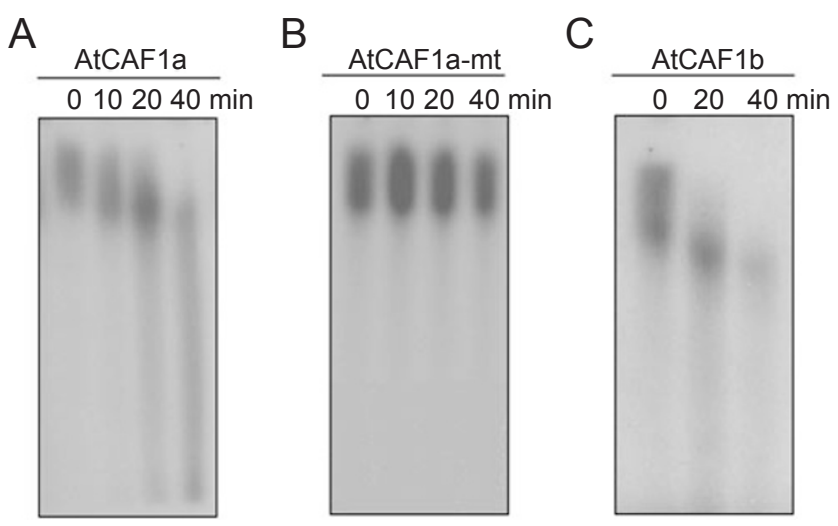

Figure 4 Nuclease activities of AtCAF1 proteins. (A) AtCAF1a shows $3^{\prime}-5$ ' exonuclease activity in vitro. (B) AtCAF1a-mt does not show exonuclease activity. (C) AtCAF1b shows exonuclease activity. For all panels, purified His-AtCAF1 fusion proteins were incubated with 5 '-labelled poly $(A)$ for the indicated time periods and the reaction mixtures were resolved on polyacrylamide gels. 
A
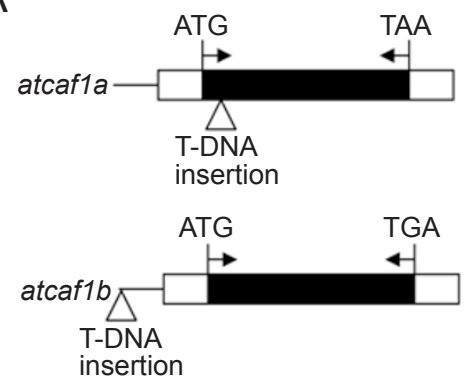
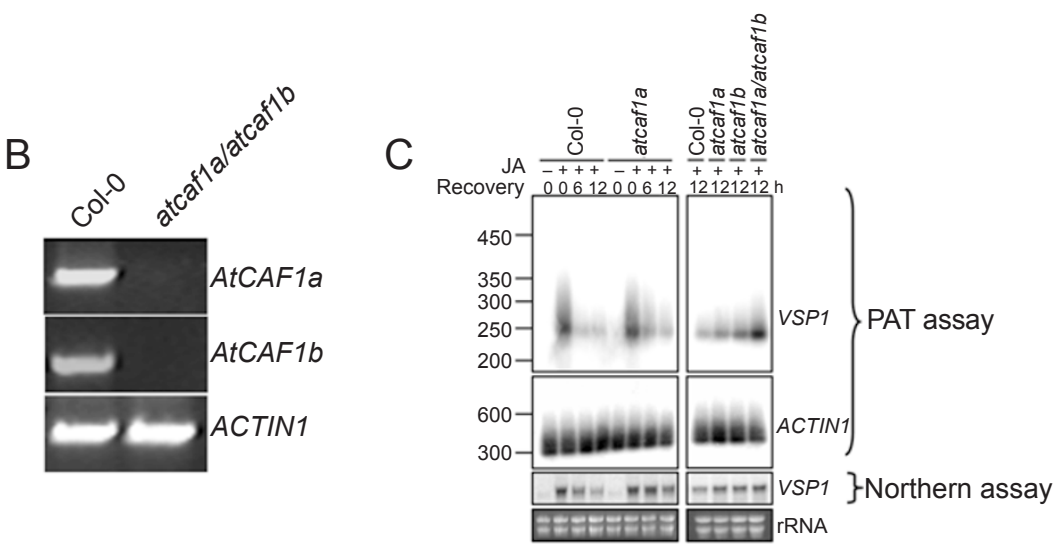
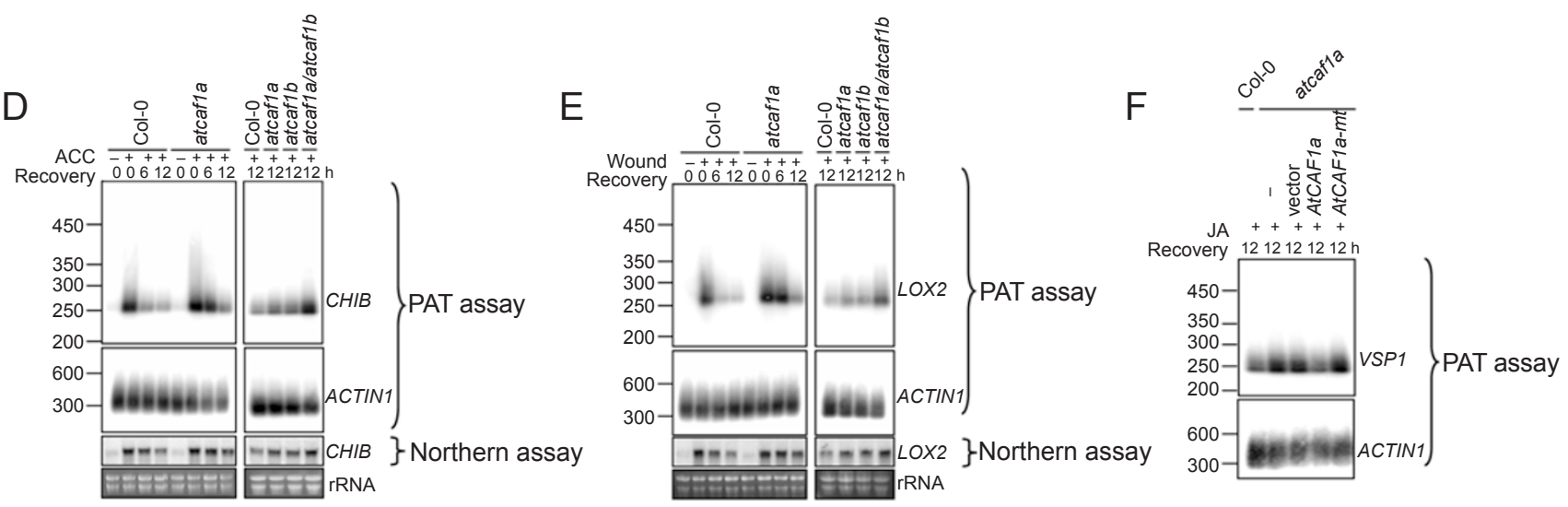

Figure 5 Loss-of-function of AtCAF1a or AtCAF1b leads to defective mRNA deadenylation. (A) Schemes of T-DNA insertion lines of each locus. Gray boxes indicate open reading frames (ORFs), and white boxes indicate untranslated regions (UTRs). Arrows indicate the relative positions of the primers used for RT-PCR analysis to check the expression of AtCAF1a or AtCAF1b in T-DNA insertion lines. Locus numbers, open reading frames, and T-DNA insertion sites are shown. (B) AtCAF1a and AtCAF1b expression in wild type and atcaf1a/atcaf1b revealed by RT-PCR analysis. Total RNAs from 2-week-old seedlings of the indicated genotypes were used as a template. Amplification of the ACTIN1 gene serves as a control. (C) PAT assays measured the deadenylation of VSP1 mRNA. Two-week-old wild-type and atcaf1a seedlings were not treated (-) or were treated with $50 \mu \mathrm{M}$ MeJA for $6 \mathrm{~h}(+)$. Plant tissues were then collected for RNA extraction at the indicated times (h) after MeJA treatment. atcaf1 $b$ and atcaf1a/atcaf1b mutants were only harvested $12 \mathrm{~h}$ after JA treatment. RNA gel blot analysis was performed to show the actual expression levels of VSP1. (D) PAT assays measured the deadenylation of CHIB mRNA. Twoweek-old wild-type and atcaf1a seedlings were not treated (-) or were treated with ACC for $24 \mathrm{~h}(+)$. Plant tissues were then collected for RNA extraction at the indicated times (h) after ACC treatment. atcaf1 b and atcaf1a/atcaf1b mutants were only harvested $12 \mathrm{~h}$ after ACC treatment. RNA gel blot analysis was performed to show the actual expression levels of $C H I B$. (E) PAT assays measured the deadenylation of LOX2 mRNA. Two-week-old wild-type and atcaf1a seedlings were not wounded $(-)$ or were wounded with a haemostat and incubated for $3 \mathrm{~h}(+)$ in a growth chamber. Plant tissues were then collected for RNA extraction at the indicated times $(\mathrm{h})$. atcaf1b and atcaf1a/atcaf1b mutants were only harvested after $12 \mathrm{~h}$. RNA gel blot analysis was performed to show the actual expression levels of LOX2. (F) Deadenylation of VSP1 mRNA in vivo is dependent on the deadenylase activity of AtCAF1a. Empty plasmid vector, wild-type AtCAF1 or AtCAF1a-mt was expressed in atcaf1a plants. Plants were harvested $12 \mathrm{~h}$ after a 6-h treatment with MeJA treatment. The deadenylation of the Arabidopsis ACTIN1 gene, which is not induced by stress treatments, was used as a control in all of the PAT assays. The positions of DNA size markers (in bp) are indicated on the left.

(Figure 4A). AtCAF1a-mt, which contains point mutations in the conserved putative catalytic residues D47/A and E49/A, completely abolished the exonuclease activity (Figure 4B). These results showed that AtCAF1a is a functional deadenylase in vitro and that its conserved DEDDh domain is required for its enzymatic activity. Similar exonuclease activity was also observed with AtCAF1b (Figure 4C). 
AtCAF $1 a$ and AtCAF1b are required for regulated $m R N A$ deadenylation

To examine whether AtCAFla plays a role in mRNA deadenylation in vivo, we determined whether atcafla, a T-DNA insertion mutant that disrupts the expression of AtCAF1a (Figure 5A and 5B), shows defective deadenylation activity. The finding that the expression of $A t$ $C A F 1 a$ was transiently induced by JA prompted us to investigate whether the atcafla mutation affects the decay of the vegetative storage protein 1 (VSPl) mRNA, which is widely used as a JA-inducible marker gene in Arabidopsis [29]. For this experiment, wild-type and atcafla plants were treated with methyl jasmonate (MeJA) for 6 $\mathrm{h}$ and then transferred to MS media for the indicated time periods (Figure 5C). Thus, a pool of newly transcribed mRNAs was produced and a transcriptional pulse-chase experiment to access the time course of deadenylation was possible. Poly (A) tail length (PAT) assays indicated that, compared with that in wild type, the deadenylation rate of $V S P 1$ mRNA was reduced in the atcafla mutant (Figure 5C). Similarly, the deadenylation rate of VSP1 mRNA was also decreased in atcaflb, a T-DNA insertion mutant that affects the expression of AtCAF1b (Figure 5A-5C). Furthermore, the impaired deadenylation rate of VSP1 mRNA was more severe in the atcaflalatcaflb double mutant than those in the single mutants or in wild type (Figure 5B and 5C). Consistent with their defective deadenylation activities, our RNA gel blot analysis indicated that the actual accumulation levels of VSP1 mRNA in the single and double mutants were higher than those in wild type (Figure 5C). These results suggested that $A t C A F 1 a$ and $A t C A F 1 b$ act redundantly in VSP1 mRNA deadenylation.

The single mutants, atcafla and atcaflb, and the double mutant were also compared with wild type for their deadenylation rates of the stress-related genes CHITINASE B (CHIB) and LIPOXYGENASE2 (LOX2). The ethylene- and JA-responsive $C H I B$ encodes a basic chitinase with antimicrobial properties $[30,31]$. LOX2 encodes a key enzyme in the octadecanoid pathway leading to JA biosynthesis [32] and is extensively used as a marker for wound responses. Results from the PAT assays showed that the mutants also showed substantially reduced deadenylation rates of CHIB (Figure 5D) and LOX2 (Figure 5E) mRNAs. In addition, RNA gel blot analyses indicated that the defective deadenylation capacities in these mutants led to increased mRNA levels of $C H I B$ and $L O X 2$ (Figure 5D and 5E). These observations indicated that $A t C A F 1 a$ and $A t C A F 1 b$ are required for regulated deadenylation of a broad spectrum of stress-responsive mRNAs. In contrast, our parallel experiments indicated that $A t C A F 1 a$ and $A t C A F 1 b$ have little effect on the deadenylation of the Arabidopsis ACTIN1 gene (Figure $5 \mathrm{C}-5 \mathrm{E}$ ), which is usually not induced by stresses.

\section{Deadenylase activity of AtCAF la is crucial for regulated} $m R N A$ deadenylation in vivo

To determine whether the exonuclease activity of AtCAF1a is required for deadenylation of VSP1 mRNA, we introduced AtCAFla or AtCAFla-mt, a mutant version of AtCAFla, into the atcafla mutant (Figure 6F and 6G). As described above, AtCAFla-mt contains two missense substitutions that inactivate the deadenylase activity of AtCAF1a (Figure 4B). PAT assays indicated that AtCAFla rescued the atcafla defect in VSP1 mRNA deadenylation, but the empty vector and AtCAFla-mt did not rescue the defect (Figure 5F). Similarly, AtCAF1a also rescued the atcafla defect in mRNA deadenylation of CHIB and LOX2 (data not shown). These results provided evidence that the enzymatic activity of AtCAF1a is essential for regulated deadenylation of mRNAs in vivo.

Over-and under-expression of AtCAFla or AtCAF1b affect plant responses to pathogen infection

The expression of AtCAF1a and AtCAF1b was upregulated after infection by $P s t$ DC3000 (Figure 1). In addition, the expression levels of AtCAF1a or AtCAF1b affected the deadenylation of several stress-responsive mRNAs. These two findings prompted us to investigate whether over- or under-expression of these genes result in altered responses of plants to pathogen infection. To this end, we generated transgenic plants showing increased expression of AtCAFla under the control of the CaMV 35S promoter (Figure 6A and 6B). The AtCAF1a over-expression line (all AtCAF1a over-expression lines showed similar results; thus, only data obtained with one line are shown below), together with the single and double mutants of AtCAF1a and AtCAF1b, were compared with wild type for their responses to Pst DC3000 infection. Quantification of pathogen growth indicated that, while the AtCAFla over-expression line showed significantly increased resistance to Pst DC3000, the single and double mutants were more susceptible to this pathogen (Figure 6C).

In agreement with the performances of the different genotypes to pathogen infection, our RT-PCR and quantitative real-time PCR analyses indicated that, compared with wild-type plants, the AtCAFla over-expression plants showed constitutively higher expression of the pathogenesis-related $(P R)$ genes $P R 1$ and $P R 2$. In contrast, the single and double mutants of AtCAFla and $A t C A F 1 b$ showed reduced expression levels of $P R 1$ and $P R 2$ (Figure 6A and 6B). Given that both AtCAF1a and AtCAF $1 \mathrm{~b}$ show deadenylation activity, we tested whether 
the two proteins act directly on the poly (A) tails of $P R 1$ and $P R 2$ transcripts. Our PAT assays indicated that the poly (A) tail levels of $P R 1$ and $P R 2$ in AtCAF1a overexpression lines were significantly higher than those in wild-type plants. In the double-mutant plants, however, the poly (A) tail levels of $P R 1$ and $P R 2$ mRNAs were much lower than those in wild-type and single-mutant plants (Figure 6D and 6E). These results are the opposite of what would be predicted if AtCAF1 proteins act directly on the deadenylation of $P R 1$ and $P R 2$. Together, our data suggest that, even though AtCAF1a and AtCAF1b are functional deadenylases, they do not act directly on the poly (A) tails of $P R 1$ and $P R 2$.

Deadenylase activity of AtCAFla is essential for the elevated expression of $P R 1$ and $P R 2$

To test whether the AtCAF1a enzymatic activity is required for $P R 1$ and $P R 2$ expression, we analysed the expression levels of $P R 1$ and $P R 2$ in atcafla mutants over-expressing AtCAFla or the above-described AtCAFla-mt. Both the wild type and the mutant version of AtCAFla were expressed at nearly the same level in the atcafla mutant background (Figure 6F and 6G). Overexpression of wild-type AtCAFla increased the expres-
A

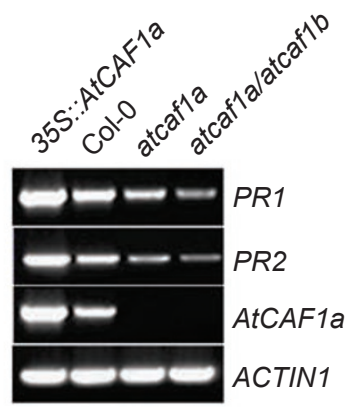

B
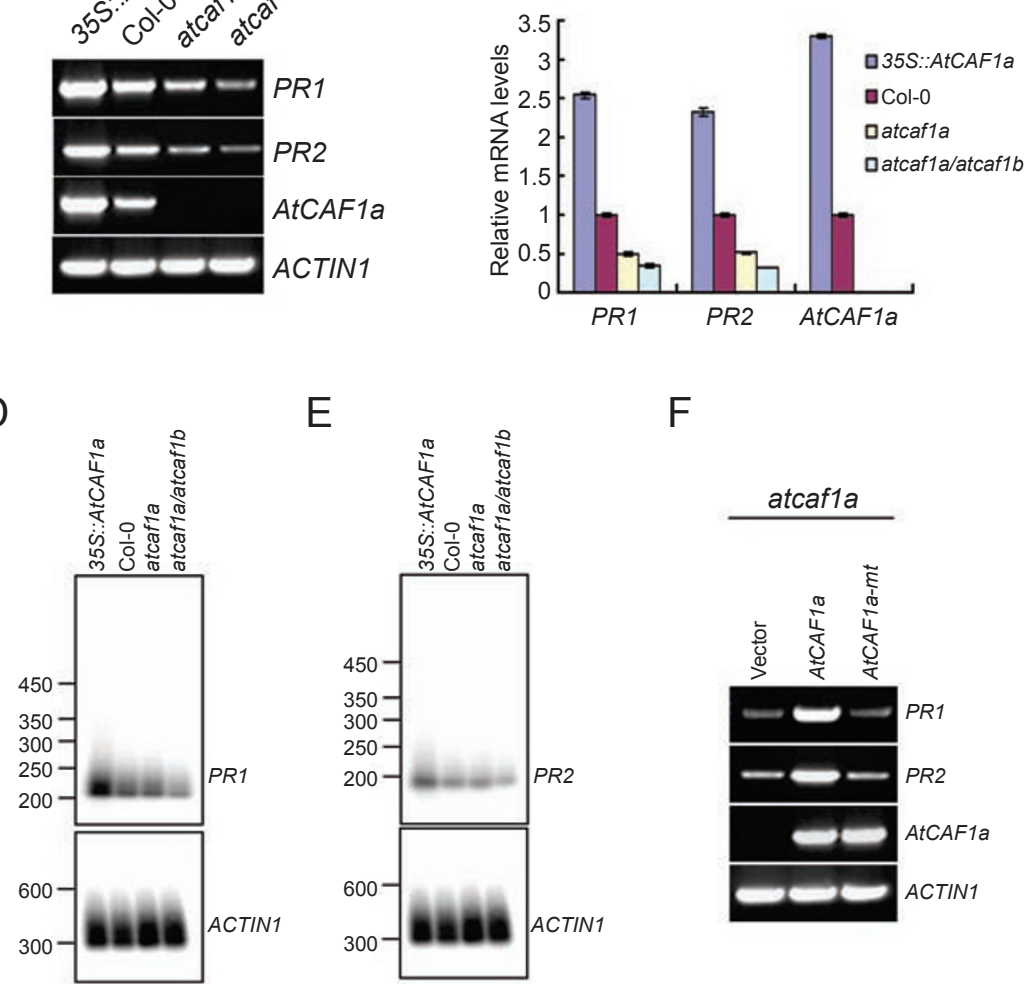

$E$

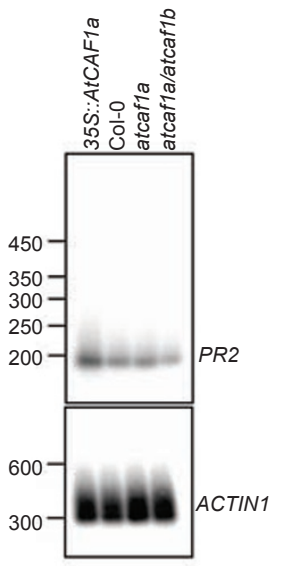

F

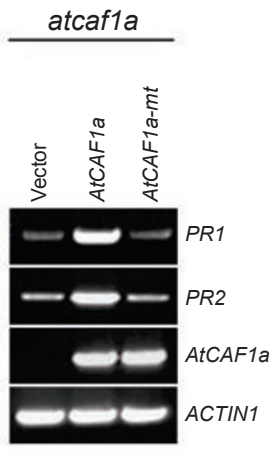

C

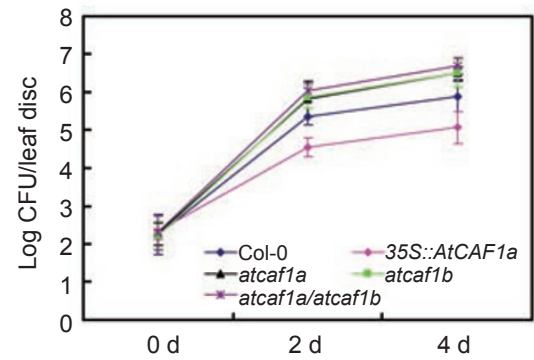

G

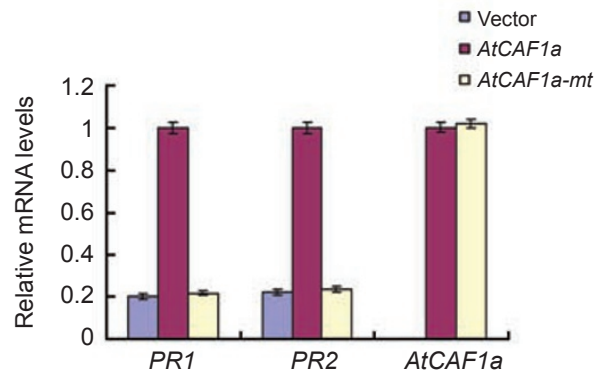

Figure 6 AtCAF1a and AtCAF1b affect plant response to Pst DC3000. (A) Steady-state expression levels of PR1 and PR2 in the indicated genotypes revealed by RT-PCR assays. Amplification of the ACTIN1 gene serves as a control. (B) Steadystate expression levels of $P R 1$ and $P R 2$ in the indicated genotypes measured by qRT-PCR assays. Transcript levels of $P R 1$ and $P R 2$ were normalised to the expression of the ACTIN1 gene measured in the same RNA samples. Data are mean \pm SD of three independent experiments. (C) Bacterial growth in wild-type, 35S::AtCAF1a, atcaf1a, atcaf1b, and atcaf1a/atcaf1b plants. The number of colony-forming units (CFU) per leaf disk was determined 0,2 , and 4 days after infiltration. Data are mean \pm SD of three replicates. (D) Deadenylation of PR1 mRNA in the indicated genotypes as revealed by PAT assay. (E) Deadenylation of PR2 mRNA in the indicated genotypes as revealed by PAT assay. For (D) and (E), 2-week-old seedlings grown under normal conditions were used for RNA extraction. Deadenylation of the ACTIN1 gene in the same RNA samples served as a control. (F) Expression levels of $P R 1$ and $P R 2$ under normal conditions in the indicated genotypes revealed by RT-PCR assays. Amplification of the ACTIN1 gene served as a control. (G) Expression levels of PR1 and PR2 under normal conditions in the indicated genotypes as measured in qRT-PCR assays. Transcript levels of $P R 1$ and $P R 2$ were normalised to the expression of the ACTIN1 gene measured in the same RNA samples. Data are mean \pm SD of three independent experiments. 
sion levels of $P R 1$ and $P R 2$, but over-expression of the mutant did not (Figure 6F and 6G). These results indicate that the enzymatic activity of AtCAF1a is important for the elevated expression of $P R 1$ and $P R 2$.

\section{Discussion}

In yeast (Saccharomyces cerevisiae), the CAF1 protein functions as one of the nine components of the CCR4-NOT complex [4, 10]. Our sequence analysis and yeast two-hybrid assays revealed that the genome of $\mathrm{Ara}$ bidopsis contains homolog(s) for all of the major components of the yeast CCR4-NOT complex except CAF130 (data not shown). We demonstrate herein that several stress-related hormones and stress stimuli (mechanical wounding and pathogen infection) induce the expression of AtCAF1a and AtCAF1b, putative Arabidopsis homologs of the yeast $C A F 1$ gene (Figure 1). Both AtCAF1a and AtCAF1b partially complement the phenotypes of the yeast cafl mutant and exhibit 3'-5' exonuclease activity in vitro (Figure 4), suggesting that they may act as functional deadenylases in vivo. We further explored the physiological significance of the deadenylation activities of the AtCAF 1 proteins in mRNA degradation in vivo. Stress-related hormones and environmental stresses induce the expression of many defence-related mRNAs, and fulfilment of these signal transduction pathways requires the removal of earlier products of gene expression. Although some of these events occur at the protein level, including the rapid proteolysis of the AUX/IAA proteins following auxin binding to the TIR1 F-box protein [33, 34], some occur at the RNA level. It is well known that JA treatment leads to increased production and stabilisation of VSP1 mRNA [29]; recovery from JA treatment, however, results in deadenylation and decay of VSP1 mRNA. Our data indicate that AtCAF1a and AtCAF1b redundantly function in regulating deadenylation of VSP1 and other stress-responsive mRNAs. Importantly, although the RNase activity is not required for the in vivo function of the yeast CAF1 protein [20], mutation in the putative catalytic residues of AtCAF1a, which abolishes its exonuclease activity in vitro, impairs the ability of mRNA deadenylation. These data support the hypothesis that the exonuclease activity of AtCAF1a is crucial for in vivo deadenylation of mRNAs.

Of interest is our findings that the expression of the At$C A F 1$ genes was induced by ABA, ACC, SA, mechanical wounding, and pathogen infection, in addition to their induction by MeJA (Figure 1). Induction of $A t C A F 1$ genes by versatile stress-related hormones and stimuli suggests that AtCAF1a and AtCAF1b may play a general role in the deadenylation of a wide range of mRNAs, rather than specifically acting on a particular stress-response pathway. It is reasonable to speculate that, whenever there is a sudden increase in mRNA production, $A t C A F 1$ genes are induced at the transcriptional level and then perform their deadenylation role on mRNAs that need to be degraded.

Emerging evidence suggested important roles for plant $C A F 1$ genes in defence responses against abiotic or biotic stresses. For example, over-expression of the pepper $C A F 1$ gene $(C a C A F 1)$ in tomato plants resulted in enhanced resistance against the oomycete pathogen, Phytophthora infestans. In addition, multiple defencerelated genes, including $P R 1$ and $P R 6$, are constitutively up-regulated in these transgenic plants [26]. One of the Arabidopsis homologs of CAF1, named CAF1-like (At$C A F 1 a$ in this study), was recently shown to be woundand biotic stress-inducible in vivo using stable transgenic lines expressing transcriptional luciferase fusions [27]

In line with these observations, our results indicated that transgenic Arabidopsis plants over-expressing At$C A F 1 a$ constitutively express higher levels of $P R 1$ and $P R 2$ and are more resistant than wild type to the infection of Pst DC3000. On the contrary, mutants of AtCAF1 genes show reduced expression of $P R 1$ and $P R 2$ and are more susceptible to the same pathogen. These results raised the interesting question of how AtCAF1a affects the expression levels of $P R 1$ and $P R 2$. Significantly, our PAT assays (Figure 6D and 6E) and complementation experiments (Figure 6F and 6G) indicate that, even though AtCAF1a does not act directly on the poly (A) tails of $P R 1$ and $P R 2$ mRNAs, its deadenylation activity is essential for the maintenance of the elevated expression levels of $P R 1$ and $P R 2$ in transgenic plants over-expressing AtCAF1a. It is likely that AtCAF1a acts indirectly in controlling $P R 1$ and $P R 2$ expression levels. We speculate that AtCAF1 proteins are required for degradation of a particular mRNA species specifying a repressor of $P R 1$ and $P R 2$ transcription. Wild-type plants, in which AtCAF1 proteins partially degrade the repressor, exhibit an intermediate level of $P R 1$ and $P R 2$ expression and disease resistance. In plants that over-express the AtCAF 1 genes, the repressor is largely or completely degraded, conferring an enhanced defence response by production of higher levels of $P R 1$ and $P R 2$. In mutants that lack the deadenylase activity of AtCAF1 proteins, the repressor is more stable and interferes with $P R 1$ and $P R 2$ expression, consequently reducing pathogen resistance. Support of this hypothesis comes from the characterisation of the Arabidopsis CER7 (WAX-DEFICIENT ECERIFERUM 7) protein, which is a putative $3^{\prime}-5^{\prime}$ exoribonuclease homologous to yeast Ribonuclease PH45 (RRP45p), a core subunit of the RNA processing and degrading exosome 
[35]. CER7 regulates cuticular wax biosynthesis, probably by degrading a specific mRNA species that encodes a negative regulator of the transcription of CER3/WAX2/ $Y R E$, a key wax biosynthetic gene [35]. Further studies are required to elucidate the detailed mechanisms of how AtCAF1 proteins affect the expression of $P R 1 / 2$ and perhaps other defence-related genes.

Disruption of genes encoding AtCAF1a or/and AtCAF $1 b$ causes no apparent developmental phenotype. This is likely because of a redundancy in mRNA deadenylation systems in Arabidopsis. AtPARN, homolog of the important deadenylase PARN in mammalian cells, has deadenylation activity on some embryo-specific transcripts [8]. Furthermore, the genome of Arabidopsis also encodes a putative homolog of yeast PAN2 protein. These two deadenylases might compensate for the AtCAF1a and AtCAF1b defects of the mutants.

\section{Materials and Methods}

\section{Oligonucleotides used in this study}

All oligonucleotides used in this study are listed in Supplementary information, Table S1. Restriction enzyme sites and point mutation sequences are underlined.

\section{Plant growth conditions}

All Arabidopsis lines used were in the Columbia (Col-0) background. Arabidopsis seeds were surface sterilised with $10 \%(\mathrm{v} / \mathrm{v})$ bleach for $10 \mathrm{~min}$ and washed five times with sterile water. Sterilised seeds were then suspended in $0.1 \%$ agarose and plated on Murashige and Skoog media. Plants were vernalised in darkness for $3 \mathrm{~d}$ at $4{ }^{\circ} \mathrm{C}$ and then transferred to a phytotrone set at $22{ }^{\circ} \mathrm{C}$ with a 16-h light/8-h dark cycle. After 2-3 weeks, seedlings were also potted in soil and placed in a growth room at $22{ }^{\circ} \mathrm{C}$ with a 16-h light/8-h dark cycle.

\section{Mutant identification and generation of transgenic plants}

Arabidopsis mutants atcafla (SALK_070336) and atcaflb (SALK_092761) were obtained from the Arabidopsis Biological Resource Center (ABRC). Homozygous T-DNA insertion lines for atcafla and atcaflb were identified with diagnostic PCR using gene-specific primers and T-DNA primers (Supplementary information, Table S1). Disruption of target gene expression in these mutants was verified by RT-PCR (Figure 5B). Mutant atcafla and atcaflb lines were crossed, and an atcafla/atcaflb double-mutant line was identified from the resulting F2 population by PCR-based genotyping.

The coding sequence of AtCAFla was amplified by PCR using the primer pairs as indicated in Supplementary information, Table $\mathrm{S} 1$. The resulting fragments were cloned into the $\mathrm{BamHI}$ and $\mathrm{SacI}$ sites of the binary vector pCanG-HA under the control of the $35 \mathrm{~S}$ promoter to generate the $35 S:: A t C A F 1 a$ construct. Similarly, we also generated a $35 S:: A t C A F 1 a-m t$ construct. Plant transformation was done with the vacuum infiltration method [36].

Plant treatments and bacterial infection

All hormones were purchased from Sigma (St Louis, MO). For hormone treatments, 2-week-old seedlings grown on MS media were treated with $50 \mu \mathrm{M}$ MeJA, $20 \mu \mathrm{M}$ ABA, $50 \mu \mathrm{M}$ ACC, or $20 \mu \mathrm{M}$ SA. For the wounding treatment, leaves of 2 -week-old plants were crushed two times across the apical lamina with a haemostat. Plants were incubated for various periods, after which tissues were harvested for RNA extraction.

Soil-grown plants that were 4-week old were infected with Pst DC3000. Bacteria were applied in a density of $10^{4} \mathrm{CFU} / \mathrm{ml}$ with a needleless syringe in the middle of a leaf, and leaf discs were cut 0 , 2 , and $4 \mathrm{~d}$ after infection. Leaf discs were cut with a cork borer and immediately homogenised with sterile water. Appropriate dilutions were plated on Kings B plates with $50 \mu \mathrm{g} / \mathrm{ml}$ rifampicin and incubated for $48 \mathrm{~h}$ at $28^{\circ} \mathrm{C}$.

\section{Gene expression analyses}

Total RNA was isolated from 14-d-old seedlings grown on MS media using a guanidine thiocyanate extraction method. Total RNA $(20 \mu \mathrm{g})$ was separated by electrophoresis on a 1\% MOPSagarose gel containing 5.8\% formaldehyde, and RNA gel blot analysis was performed as described previously [28]. For RT-PCR, $5 \mu \mathrm{g}$ of total RNA was used for first-strand cDNA synthesis by M-MLV (Promega). PCR conditions were as follows: $3 \mathrm{~min}$ at $94{ }^{\circ} \mathrm{C}$, followed by 30 cycles of $30 \mathrm{~s}$ at $94{ }^{\circ} \mathrm{C} ; 30 \mathrm{~s}$ at $55^{\circ} \mathrm{C}$; and 1 min at $72{ }^{\circ} \mathrm{C}$. Quantitative real-time RT-PCR (qRT-PCR) analyses were performed using the QuantiTec SYBR green system (Qiagen) and the Opticon PCR machine (MJ Research, Waltham, MA). Data were treated using the Opticon Monitor 3 software provided by the manufacturer. Gene-specific primers used for RT-PCR and qRTPCR assays are listed in Supplementary information, Table S1.

To express GUS under the control of Arabidopsis native promoters, 2628-bp and 1456-bp regions of 5' upstream sequences of AtCAF1a and AtCAF1b, respectively, were amplified from the relevant genomic regions. These promoter fragments were fused to a GUS coding sequence and transformed into wild-type Arabidopsis plants. Histochemical staining for GUS activity in transgenic plants was performed as described previously [37].

\section{Site-directed mutagenesis of AtCAFla}

Point mutations of AtCAFla were done with the MutantBEST Kit (TaKaRa) according to the manufacturer's instructions.

\section{Yeast complementation}

Yeast strains KY803 (MATa leu2-PET56 trp1- 1 ura3-52 gal2 gcn4- $\Delta 1$ ) and KY803-c1 (MATa leu2-PET56 trp1- 1 l ura3-52 gal2 gcn4- $\Delta 1$ cafl $:$ LEU2) were kindly provided by Clyde L. Denis. Yeast strains were grown on YEP medium (1\% yeast extract, $2 \%$ Bacto peptone) supplemented with $2 \%$ glucose. YD plates consisted of YEP media supplemented with $2 \%$ glucose and $2 \%$ agar. The open reading frames (ORFs) of $A t C A F 1$ genes were cloned into pYES2, and yeast complementation studies were done according to Ohn et al. [21].

\section{Nuclease assay}

For the expression of AtCAF1 proteins as fusions with the His tag, the open reading frames of $A t C A F 1$ genes were cloned into pET-28a $(+)$ (Novagen). Expression of His-AtCAF1 fusions was carried out in BL21 (DE3) host strains, and purification was done with Ni-NPA according to the manufacturer's instructions (Novagen). In vitro $\mathrm{RNase}$ assays were performed in $20 \mathrm{mM}$ Tris/ $\mathrm{Cl}(\mathrm{pH}$ 
7.0), $150 \mathrm{mM} \mathrm{NaCl}, 2 \mathrm{mM} \mathrm{MgCl}$, $5 \mathrm{U}$ RNasin (Promega), $1 \mathrm{mM}$ poly (A) substrate (Amersham) labelled with ${ }^{32} \mathrm{P}$ at the $5^{\prime}$ end, and $0.1 \mu \mathrm{g}$ of purified His-AtCAF1 fusion proteins. A volume of $10 \mu \mathrm{l}$ of the reaction mixture was incubated at $25{ }^{\circ} \mathrm{C}$ for the indicated time. Reactions were stopped by the addition of formamide/EDTA buffer and then loaded onto $7 \mathrm{M}$ urea/10\% acrylamide (19:1) gels [19].

\section{PAT assay}

For PAT assays, 2-week-old plants were treated with $50 \mu \mathrm{M}$ MeJA for $6 \mathrm{~h}, 50 \mu \mathrm{M}$ ACC for $24 \mathrm{~h}$, or wounded with a haemostat and incubated for $3 \mathrm{~h}$, after which plant tissues were harvested at various times for RNA extraction. PAT assays were carried out according to the method of Sallés et al. [38] with minor modifications. Briefly, $2 \mu \mathrm{g}$ of total RNA was used for RT with an anchoring nucleotide-fused oligo (dT) ${ }_{15}$ primer. PCR was performed with the anchor primer and a sense primer VSP1-mF (CHIB-mF/LOX2$\mathrm{mF}$ ) targeting a specific sequence in the cDNA of interest. PCR cycles were as follows: $3 \mathrm{~min}$ at $94{ }^{\circ} \mathrm{C}$, followed by 25 cycles of $30 \mathrm{~s}$ at $94{ }^{\circ} \mathrm{C} ; 30 \mathrm{~s}$ at $60{ }^{\circ} \mathrm{C} ; 1 \mathrm{~min}$ at $72{ }^{\circ} \mathrm{C}$. The PCR products were resolved on $2 \%$ agarose gels and subjected to Southern blot analysis.

\section{Acknowledgments}

We thank Clyde L Denis (University of New Hampshire, USA) for providing the yeast strains, Jianmin Zhou (National Institute of Biological Sciences, China) for providing the bacterial strain Pseudomonas syringae pv tomato DC3000, and Akira Sakai (Mitsubishi Kasei Institute of Life Sciences, Japan) for his insightful comments on the yeast complementation assays. We are grateful to the anonymous reviewers for their valuable suggestions for this manuscript. This work was supported by grants from the National Natural Science Foundation of China (30425033, 30530440), the Ministry of Science and Technology of China (2006CB102004, 2006AA10A116) and the Chinese Academy of Sciences (KSCX2-YW-N-045).

\section{References}

1 Tucker M, Parker R. Mechanisms and control of mRNA decapping in Saccharomyces cerevisiae. Annu Rev Biochem 2000; 69:571-595.

2 Parker R, Song H. The enzymes and control of eukaryotic mRNA turnover. Nat Struct Mol Biol 2004; 11:121-127.

3 Cao D, Parker R. Computational modeling of eukaryotic mRNA turnover. RNA 2001; 7:1192-1212.

4 Denis CL, Chen J. The CCR4-NOT complex plays diverse roles in mRNA metabolism. Prog Nucleic Acid Res Mol Biol 2003; 73:221-250.

5 Brown CE, Sachs AB. Poly (A) tail length control in Saccharomyces cerevisiae occurs by message-specific deadenylation. Mol Cell Biol 1998; 18:6548-6559.

6 Hammet A, Pike BL, Heierhorst J. Posttranscriptional regulation of the RAD5 DNA repair gene by the Dun1 kinase and the Pan2-Pan3 poly (A)-nuclease complex contributes to survival of replication blocks. J Biol Chem 2002; 277:22469-22474.

7 Gao M, Fritz DT, Ford LP, Wilusz J. Interaction between a poly (A)-specific ribonuclease and the 5' cap influences mRNA deadenylation rates in vitro. Mol Cell 2000; 5:479-488.

8 Reverdatto SV, Dutko JA, Chekanova JA, Hamilton DA, Belostotsky DA. mRNA deadenylation by PARN is essential for embryogenesis in higher plants. RNA 2004; 10:1200-1214.

9 Nishimura N, Kitahata N, Seki M, et al. Analysis of $A B A$ hypersensitive germination 2 revealed the pivotal functions of PARN in stress response in Arabidopsis. Plant J 2005; 44:972984.

10 Collart MA, Timmers HT. The eukaryotic Ccr4-not complex: a regulatory platform integrating mRNA metabolism with cellular signaling pathways? Prog Nucleic Acid Res Mol Biol 2004; 77:289-322.

11 Liu HY, BadarinarayanaV, Audino DC, et al. The NOT proteins are part of the CCR4 transcriptional complex and affect gene expression both positively and negatively. EMBO J 1998; 17:1096-1106.

12 Bai Y, Salvadore C, Chiang YC, et al. The CCR4 and CAF1 proteins of the CCR4-NOT complex are physically and functionally separated from NOT2, NOT4, and NOT5. Mol Cell Biol 1999; 19:6642-6651.

13 Chen J, Rappsilber J, Chiang YC, Russell P, Mann M, Denis CL. Purification and characterization of the 1.0 MDa CCR4NOT complex identifies two novel components of the complex. J Mol Biol 2001; 314:683-694.

14 Tucker M, Valencia-Sanchez MA, Staples RR, Chen J, Denis CL, Parker R. The transcription factor associated Ccr4 and Cafl proteins are components of the major cytoplasmic mRNA deadenylase in Saccharomyces cerevisiae. Cell 2001; 104:377386.

15 Chen J, Chiang YC, Denis CL. CCR4, a 3'-5' poly (A) RNA and ssDNA exonuclease, is the catalytic component of the cytoplasmic deadenylase. EMBO J 2002; 21:1414-1426.

16 Tucker M, Staples RR, Valencia-Sanchez MA, Muhlrad D, Parker R. Ccr4p is the catalytic subunit of a Ccr4/Pop2p/Notp mRNA deadenylase complex in Saccharomyces cerevisiae. EMBO J 2002; 21:1427-1436.

17 Daugeron MC, Mauxion F, Séraphin B. The yeast POP2 gene encodes a nuclease involved in mRNA deadenylation. Nucleic Acids Res 2001; 29:2448-2455.

18 Zuo Y, Deutscher MP. Exoribonuclease superfamilies: structural analysis and phylogenetic distribution. Nucleic Acids Res 2001; 29:1017-1026.

19 Thore S, Mauxion F, Séraphin B, Suck D. X-ray structure and activity of the yeast Pop2 protein: a nuclease subunit of the mRNA deadenylase complex. EMBO Rep 2003; 4:1150-1155.

20 Viswanathan P, Ohn T, Chiang YC, Chen J, Denis CL. Mouse CAF1 can function as a processive deadenylase $/ 3^{\prime}-5^{\prime}$ exonuclease in vitro but in yeast the deadenylase function of CAF1 is not required for mRNA poly(A) removal. J Biol Chem 2004; 279:23988-23995.

21 Ohn T, Chiang YC, Lee DJ, Yao G, Zhang CX, Denis CL. CAF1 plays an important role in mRNA deadenylation separate from its contact to CCR4. Nucleic Acids Res 2007; 35:3002-3015.

22 Sakai A, Chibazakura T, Shimizu Y, Hishinuma F. Molecular analysis of $P O P 2$ gene, a gene required for glucose-derepression of gene expression in Saccharomyces cerevisiae. Nucleic Acids Res 1992; 20:6227-6233.

23 Hata H, Mitsui H, Liu H, et al. Dhh1p, a putative RNA heli- 
case, associates with the general transcription factors Pop2p and Ccr4p from Saccharomyces cerevisiae. Genetics 1998; 148:571-579.

24 Berthet C, Morera AM, Asensio MJ, et al. CCR4-associated factor CAF1 is an essential factor for spermatogenesis. Mol Cell Biol 2004; 24:5808-5820.

25 Molin L, Puisieux A. C. elegans homologue of the Cafl gene, which encodes a subunit of the CCR4-NOT complex, is essential for embryonic and larval development and for meiotic progression. Gene 2005; 358:73-81.

26 Sarowar S, Oh HW, Cho HS, et al. Capsicum annuum CCR4associated factor $C a C A F 1$ is necessary for plant development and defence response. Plant J 2007; 51:792-802.

27 Walley JE, Coughlan S, Hudson ME, et al. Mechanical stress induces biotic and abiotic stress responses via a novel ciselement. PLoS Genet 2007; 3:1800-1812.

28 Zheng W, Zhai Q, Sun J, et al. Bestatin, an inhibitor of aminopeptidases, provides a chemical genetics approach to dissect jasmonate signaling in Arabidopsis. Plant Physiol 2006; 141:1400-1413.

29 Berger S, Bell E, Mullet JE. Two methyl jasmonate-insensitive mutants show altered expression of AtVsp in response to methyl jasmonate and wounding. Plant Physiol 1996; 111:525-531.

30 Samac DA, Hironaka CM, Yallaly PE, Shah DM. Isolation and characterization of the genes encoding basic and acidic chitinase in Arabidopsis thaliana. Plant Physiol 1990; 93:907-914.

31 Lorenzo O, Piqueras R, Sánchez-Serrano JJ, Solano R. ETHYLENE RESPONSE FACTOR1 integrates signals from ethylene and jasmonate pathways in plant defense. Plant Cell 2003; 15:165-178.

32 Bell E, Creelman RA, Mullet JE. A chloroplast lipoxygenase is required for wound-induced jasmonic acid accumulation in Arabidopsis. Proc Natl Acad Sci USA 1995; 92:8675-8679.

33 Dharmasiri N, Dharmasiri S, Estelle M. The F-box protein TIR1 is an auxin receptor. Nature 2005; 435:441-445.

34 Kepinski S, Leyser O. The Arabidopsis F-box protein TIR1 is an auxin receptor. Nature 2005; 435:446-451.

35 Hooker TS, Lam P, Zheng H, Kunst L. A core subunit of the RNA-processing/degrading exosome specifically influences cuticular wax biosynthesis in Arabidopsis. Plant Cell 2007; 19:904-913.

36 Bechtold N, Pelletier G. In planta Agrobacterium-mediated transformation of adult Arabidopsis thaliana plants by vacuum infiltration. Methods Mol Biol 1998; 82:259-266.

37 Jefferson RA. Assaying chimeric genes in plants: The GUS gene fusion system. Plant Mol Bio Rep 1987; 5:387-405.

38 Sallés FJ, Richards WG, Strickland S. Assaying the polyadenylation state of mRNAs. Methods 1999; 17:38-45.

(Supplementary information is linked to the online version of the paper on the Cell Research website.) 